\title{
Low-energy nuclear reactions and the leptonic monopole
}

\section{Georges Lochak*, Leonid Urutskoev**}

*Fondation Louis de Broglie, Paris, France

**RECOM, Kurchatov Institute, Moscow, Russia

\section{Transformation}

Our report surveys the experimental and theoretical studies carried out at the RECOM since 1998 and the theoretical studies of leptonic monopoles by Georges Lochak (Fondation Louis de Broglie). We will outline briefly all the results to give the overall picture of our research.

In 1998, to solve some applied problem, our research group studied the electric explosion of titanium foil in water. By pure accident, in mass-spectrometric analysis of the titanium powder formed after the electric explosion, we noted a pronounced distortion of the natural isotope composition of titanium. The principle of the experiment was as follows. Two banks of capacitors with the total energy store $\mathbf{W}=\mathbf{5 0} \mathbf{k J}$ and the voltage $\mathbf{U}=\mathbf{5} \mathbf{k V}$ are discharged simultaneously and independent of each other to two foil loads over time $\mathbf{t} \sim \mathbf{0 . 1}$ ms. Of course, during the long period of our studies, we employed different experimental block diagrams, which we cannot describe in detail here. The most general experimental diagram is shown in Fig. 1.

This figure shows a half of the setup. The load is located in the explosion chamber, which is a strong, leak-tight metal container, with an internal structure made of high-density polyethylene. The design of the explosion chamber includes facilities for the gas exhaust and bleeding-in, and for taking gas samples into cylinders. The electrodes were made of highpurity titanium. As the operating fluid, we used either bidistilled water with an impurity level of $10^{-6} \mathrm{~g} / 1$, or solutions of various metal salts in bidistilled water. 


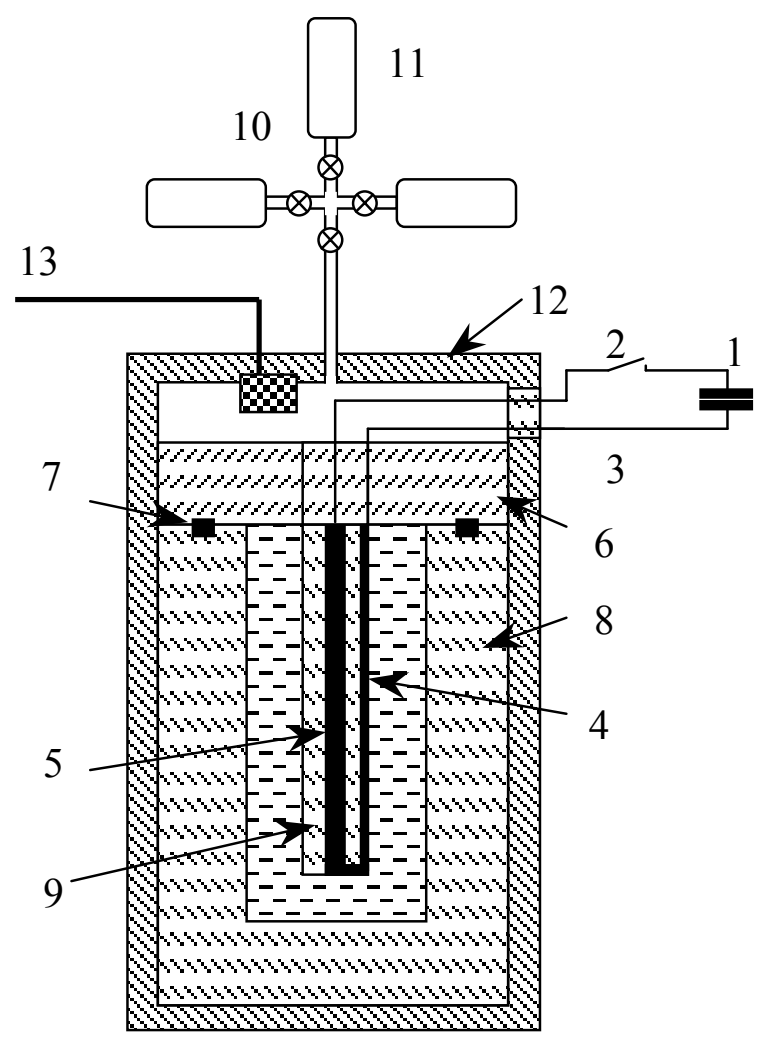

Figure 1.

Design of the experimental setup;

1. bank of capacitors; 2 . discharger;

3. wire; 4. foil; 5. electrode;

6. polyethylene cover; 7. sealing;

8. blasting chamber; 9. liquid;

10. valves; 11. gas cylinders;

12. stainless-steel shell;

13. pressure gauge

The key result is as follows. The remainder of the titanium foil shows a distorted titanium isotope ratio (Fig. 2). It can be seen from the figure that the situation looks as if ${ }^{48} \mathrm{Ti}$ "disappeared" during the instance of the pulse. Please note that the ${ }^{48} \mathrm{Ti}$ isotope was not transformed into another isotope, but instead it disappeared, while other isotopes remained approximately in the same proportion, to within the error of measurements of course. The deficiency of ${ }^{48} \mathrm{Ti}$ in some experiments is $\sim 5 \%$ while the measurement error is $\pm 0.4 \%$. Simultaneously with disappearance of ${ }^{48} \mathrm{Ti}$, a sharp (ten-fold) increase in the impurity content in the samples was detected by mass-spectrometry, X-ray fluorescence analysis, and other methods. The percentage of the new impurities corresponded to the percentage of the lost ${ }^{48} \mathrm{Ti}$. The chemical composition of the resulting foreign components is shown in Fig. 3. All the components that could be present from the beginning have been subtracted. 


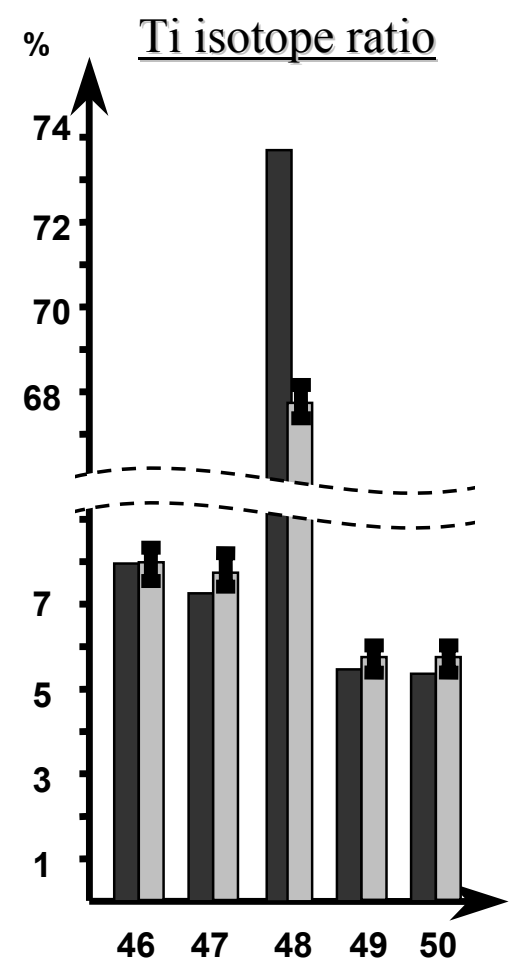

Figure 2.

Distorted titanium isotope ratio

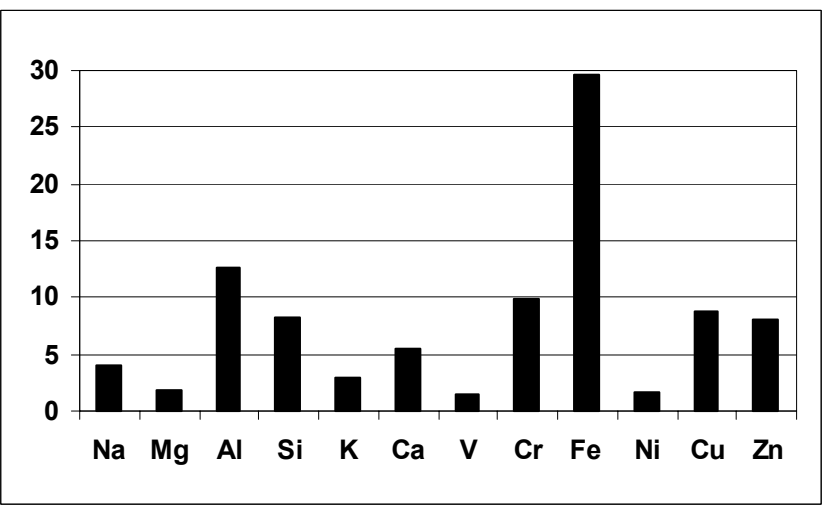

Figure 3.

The chemical composition of the resulting foreign components

I am not going to analyze the experimental results, as this analysis has been published in [1]. Nevertheless, the results were so unexpected that they called for an independent verification. This was done by our colleagues from Dubna (Kuznetsov's group). The verification was thorough, and the results were published in [2]. An important result is that, 
unlike Fleischmann and Pons, we claim that no neutrons are observed in our experiments within the limitations on a neutron flux of $\mathbf{I}<\mathbf{1 0}^{\mathbf{3}}$ per pulse. This is important evidence supporting the assumption that our "magical" nuclear transformations do not involve strong interactions.

Another important fact was found both in our experiments and in Kuznetsov's group experiments. Neither of us observed any significant residual $\gamma$-activity in the samples. The lack of excited nuclei is important because this allows one to reject all hypothetical accelerative mechanisms for the observed nuclear transformations, because it is impossible to overcome the Coulomb barrier through an acceleration mechanism without exciting the nucleus. Similarly one cannot conquer a fortress by a forward storm without destroying the walls or gates. Nevertheless, the fortress has been conquered, as follows from the experiments. Hence, we should look for evidence that the wall was undermined.

The reader may wonder how reliable these results are. Could there be a procedural mistake? The answer is as follows: the data on the isotope shift were obtained independently on three types of mass spectrometers. Hence, it is not a systematic error made on one particular type mass spectrometer. Could this isotope shift result from an error inherent in mass spectrometry, for example, from a superimposition of lighter masses? Our colleagues from Dubna verified our results on the isotope shift of ${ }^{48} \mathrm{Ti}$ using gamma-activation analysis. The idea was that nuclear transitions for titanium isotopes differ appreciably from one another and, therefore, they are easily detectable. As noted earlier, they got the same result - a loss of ${ }^{48} \mathrm{Ti}$. Thus, a procedural error also can be ruled out.

We focus on the isotope shift in detail because it proves that low-energy nuclear reactions did, in fact occur. The mere detection of foreign chemical elements in a tightly sealed chamber could be attributed to admixtures of the materials that make up the device. Conversely, an isotope shift from the natural isotope ratio of a chemical element cannot be attributed to admixtures. Indeed, the admixtures must also obey the natural isotope ratio. An occasional isotope redistribution in a sample, first, contradicts the Second Law of Thermodynamics, because it implies a spontaneous decrease in the entropy. Second, the sample is thoroughly stirred prior to mass spectrometry and a random portion is taken every time. Therefore, we would have observed not only a decrease in the ${ }^{48} \mathrm{Ti}$ content in some samples, but also an increase in this content in other samples. However, this is not the case. Last year, at the colloquium in Paris organized by Fondation Louis de Broglie, a French colleague made a felicitous remark on this topic. He said: it looks as if an isotope revolution was going to overwhelm physics. If this is really the case, please, do not imagine that Russians or Frenchmen are again responsible for a revolution. This is not at all the case; this revolution is obviously international.

Let us turn again to the results of measurements shown in Fig. 3. I intentionally did not mark the errors in the chart, as this would complicate understanding. The problem is that the accuracy of measurement is variable for different chemical elements. In some cases, it is $5 \%$; in other cases, $10 \%$, and for light elements the error may reach $30 \%$. However, this is not very important at this stage. We can even consider this picture (Figure 3) semi-quantitative. Another fact is important, namely, one isotope (the parent atom), in particular: ${ }^{48} \mathrm{Ti}$ is not 
converted into one or two daughter isotopes of another chemical element or titanium, as would be expected from the views of known nuclear physics. Instead, it decomposes into a spectrum of daughter elements. This fact by itself suggests that we are dealing with a collective process, because one atom cannot decompose into a spectrum of other atoms, if for no other reason because of the substantial difference in the nuclear binding energy.

Nevertheless, some words should be said about the procedures used to determine the composition of daughter chemical elements presented in Fig. 3. Apart from mass spectrometry, X-ray fluorescence analysis and optical spectrometry methods were used for this purpose. All the results are in satisfactory quantitative agreement. I will not dwell on this point, as it has been described in detail in $[1,2]$.

Yet another problem, namely, the reproducibility of experiments, is critical for the whole cold nuclear synthesis, and we will consider it here. The scatter of the electrical parameters of our setup (the current and the charge) from one experiment to another is about $15 \%$ with the same load. This is a good result. The usual scatter for pulsed plasma devices is about $25 \%$. We achieved a reproducibility of measurable physical parameters, for example, the isotope distortion of titanium, equal to $\pm 0.56 \%$ and the pressure jump $\delta \mathbf{P} / \mathbf{P}$, equal to $\sim 1.1 \%$. Thus, the reproducibility of physical parameters is better than that of electrical parameters, and we conclude that our experiments show a rather high reproducibility. However, we must admit that in some cases, the effect of, for example, isotope distortion increases several-fold for unknown reasons. These experiments are not reproducible, which may be due to some cosmological factor. This only means that our understand the low-energy nuclear transformations is still inadequate. By the way, the charts shown here (Fig. 2 and 3) do not include these "Olympic records."

Despite all our reasoning, one can state that an effect actually exists if a parameter has been found whose change induces an enhancement of the effect. In this particular case, the isotope shift. We were able to find such a parameter. If glycerol is added to the bidistilled water, the titanium 48 -isotope shift increases. Figure 4 shows the average ${ }^{48} \mathrm{Ti}$ isotope shifts for two series of experiments, one carried out in bidistilled water and the other, in glycerol. It can be seen from the figure that the shift is greater in glycerol. The reason for this is still unclear. 


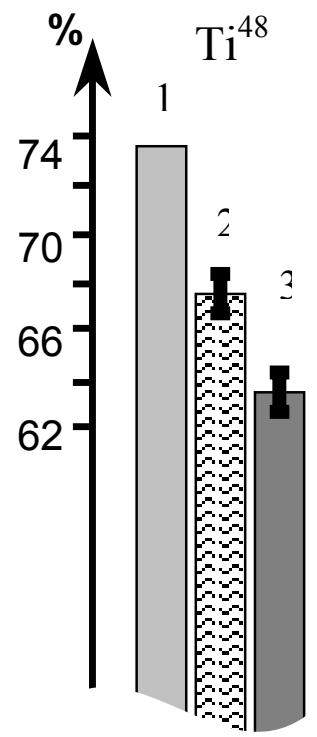

Figure 4.

Average ${ }^{48} \mathrm{Ti}$ isotope shifts for two series of experiments

1. Natural;

2. $\mathrm{H}_{2} \mathrm{O} ; 3 . \mathrm{C}_{3} \mathrm{H}_{8} \mathrm{O}_{3}$

$$
\mathbf{R}=\left(\frac{\mathbf{U}^{235}}{\mathbf{U}^{238}}\right) /\left(\frac{\mathbf{U}^{235}}{\mathbf{U}^{238}}\right)_{\text {Initial }}
$$

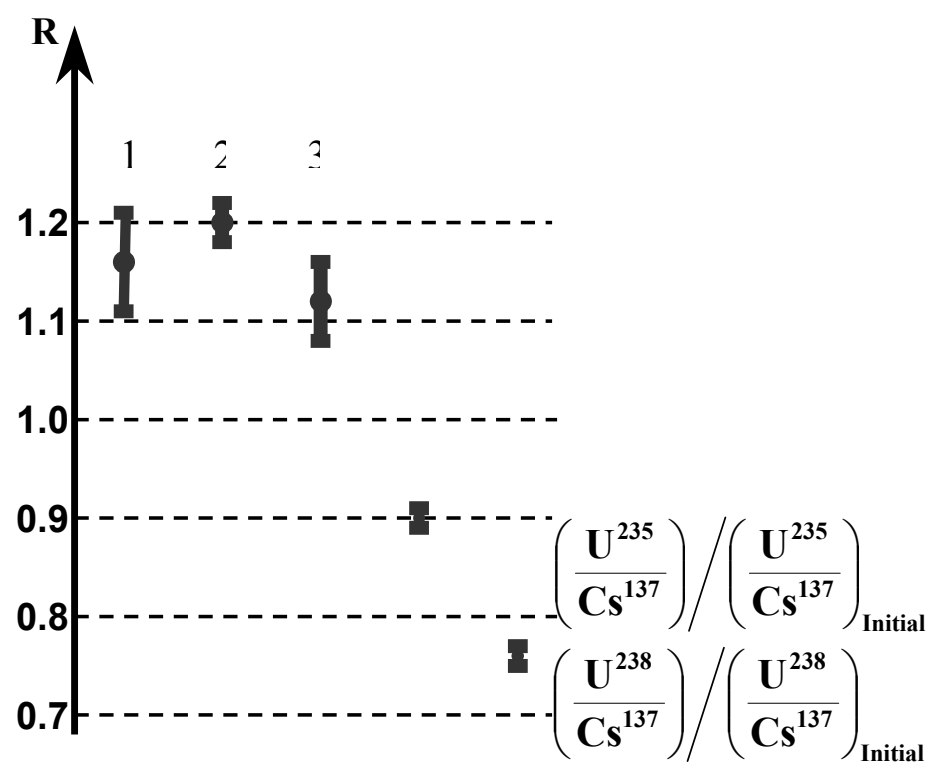

Figure 5.

The ${ }^{235} \mathrm{U} /{ }^{238} \mathrm{U}$ ratios measured by various procedures and referred to the same ratio measured in the starting solution: 1. $\alpha$-spectrometry; 2. $\gamma$ - spectrometry

3. mass - spectrometry 
A rather interesting question is whether $\mathrm{Ti}$ is the only element to possess this remarkable feature. The answer is no. Experiments with other types of foils $(\mathrm{Pb}, \mathrm{Zr}$, Ta and so on) were carried out, and isotope shifts were again detected. For example, the ${ }^{208} \mathrm{~Pb}$ isotope is the parent atom for $\mathrm{Pb}$. It is noteworthy that the tendency for transformation is usually found for even-even nuclei. Note that this is only an observation rather than a statement. We did not carry out systematic studies with other foils, but we concentrated mainly on Ti. Note that to attain significant effects in these experiments, it is necessary to carefully select the current, the weight of the load, and other parameters for each type of foil.

Nevertheless, the data obtained are sufficient to claim that each chemical element is transformed to give its own spectrum of chemical elements.

The question of the isotope ratios of the chemical elements formed upon the transformation also cannot be passed over in silence.

For the vast majority of chemical elements, we did not notice significant distortions of the isotope composition with respect to the natural distribution. This offers hope that we have not invented or imagined anything but only came across a natural phenomenon.

In order to complete the story of transformation, we should consider this problem: where does the transformation take place, either throughout the whole space of the explosion chamber or only in the plasma channel? To answer this question, we carried out experiments with uranium salts (uranyl sulfate, $\mathrm{UO}_{2} \mathrm{SO}_{4}$ ) [3].

The idea of the experiment was as follows. The plasma channel has a small volume with respect to the volume of the whole chamber. Thus, if some salt of a metal having several isotopes is added to bidistilled water, the number of admixture atoms from the solution that get to the plasma channel would be small compared to the number of Ti atoms. It is clear that recording of the isotope shift of admixture atoms would indicate that transformation takes place throughout the whole bulk of the chamber. As this metal, we used U. Uranium has two isotopes, ${ }^{235} \mathrm{U}$ and ${ }^{238} \mathrm{U}$, whose ratio can be easily measured even at a low specific concentration by means of $\gamma, \beta$ and $\alpha$-spectrometry. Figure 5 shows the ${ }^{235} \mathrm{U} /{ }^{238} \mathrm{U}$ ratios measured by various procedures and compared to the ratio measured in the starting solution. Thus, if no changes were detected after the experiment, this ratio would be equal to unity. It can be seen from the figure that the real ratio is far from unity. The isotope shift effect extends far beyond the possible errors. The shift occurs toward enrichment of the mixture in the ${ }^{235} \mathrm{U}$ isotope. This does not mean that ${ }^{238} \mathrm{U}$ is converted into ${ }^{235} \mathrm{U}$. This interpretation is wrong. We added some ${ }^{137} \mathrm{Cs}$ isotope as the marker. Then we measured the specific activity (that is, activity divided by the volume) of each $U$ isotope with respect to the Cs activity before and after the experiment. It was found that the activity of both $U$ isotopes decreased with respect to that of Cs. However, the activity of the ${ }^{238} \mathrm{U}$ isotope decreases to a greater extent. Thus, the ratio of ${ }^{235} \mathrm{U}$ to ${ }^{238} \mathrm{U}$ becomes bigger than unity. Prior to these experiments, we made sure that the specific activity of ${ }^{137} \mathrm{Cs}$ does not change noticeably. The real situation is more complicated [3] but this is a topic of a separate report. For us, it is important that the transformation can also take place outside the plasma channel. This is a rather "unpleasant surprise," because, probably, within several years, when the low-temperature transmutation will be studied in more detail, it would be rather easy to devise a facile and inexpensive 
process to enrich uranium. In view of the growth of terrorism all over the world, this outcome seems deplorable.

Here is the final remark concerning the experimental study of the transformation with regard to gases. Gases are also chemical elements, and it is likely that they are formed in these experiments. This aspect will be considered in [4].

\section{Phenomenological model}

A direct clue to the phenomenological model comes from the proportionality between the ${ }^{48} \mathrm{Ti}$ isotope shift and the percentage of foreign chemical elements observed in the experiment. Thus, a material balance equation is required. We were able to compose the balance equations for the binding energy and baryon, electric, and lepton charges [5, 6].

The first step toward the computer simulation of the low-temperature transformations was made by our colleague Doctor Penkov from Dubna [2]. We are also working along this line. Doctor Fillippov will make a detailed report on this topic [7]. We will only briefly mention the main principles that underlie the phenomenological model. We will proceed from the fact that the transformation does take place but we do not understand how the Coulomb barrier is overcome. We consider the assembly of atoms in the initial state and in the transformed state and require that all conservation laws including the energy and the baryon, electric, and lepton charge conservation laws, are fulfilled. By the way, a similar strategy was used by W. Heisenberg and M. Born in the early construction of quantum mechanics. As mentioned above, the experiment does not show the presence of radioactivity or neutrons, hence, the transformation does not involve strong interactions. Then let only weak interactions be allowed, that is, $\beta$-decay and $\mathrm{K}$-capture and the corresponding processes for positrons $\beta^{+}$. It follows from the experiment that no substantial heat evolution is observed during the transformation. This means that we must look for similar binding energies for the initial and final assemblies of atoms. Then we must decide what particular atoms are to be included in the initial assembly. It is clear that this would be atoms of the chemical elements that occur in the explosion chamber, in particular, oxygen, titanium and hydrogen. It only remains now to put a question to the Mendeleev Periodic Table and to authorize a computer to carry on a long dialog with the Table. Doctor Fillipov managed to teach a computer to do this. He will describe his experience in detail [7]. We will cite only one example

$$
{ }_{23}^{51} \mathrm{~V}+{ }_{22}^{48} \mathrm{Ti}+{ }_{8}^{18} \mathrm{O} \rightarrow{ }_{26}^{57} \mathrm{Fe}+{ }_{11}^{23} \mathrm{Na}+{ }_{17}^{37} \mathrm{Cl}+\mathrm{e}+\mathrm{o}(1 \mathrm{keV})
$$

Now, when the answer has been found, the problem looks simple and can be verified using a reference book on the nuclear binding energies. A surprising fact is that with a nuclear binding energy of about $10 \mathrm{meV}$ per nucleon, it is possible to select combinations of atoms that ensure that the total binding energies in the left and right parts of the equation coincide to within approximately $\Delta=100 \mathrm{eV}$. An even more surprising fact is that these combinations coincide qualitatively with the experimental results. For example the model including titanium, oxygen and hydrogen does not give any combinations with elements higher than zinc. This is in line with the results presented in Fig. 3. Moreover, the model predicts that the 
addition of vanadium should yield the ${ }^{57} \mathrm{Fe}$ isotope. This result was actually obtained in experiments.

We drew the following conclusions from the numerical experiment:

1. Contrary to the opinion of the majority of physicists, the possibility of lowenergy transformation does not contradict the conservation laws.

2. This process is collective in principle and can be simulated within the framework of processes based on weak interactions.

3. Since weak interactions are characterized by small cross-sections, a catalyst is needed.

\section{Monopole as a catalyst?}

Experimental searches for the monopole started immediately after the transformation phenomenon had been found. In the first experiment, the explosion chamber was insufficient sealed and the unit did not include a prechamber. Plasma broke through the sealing, and a plasma glow appeared above the unit [1]. Figure 6 shows sample photos of this, made using electron-optical image converters with an exposure time of the order of $10^{-4} \mathrm{~s}$. The time delay between the frames equals $10^{-3} \mathrm{~s}$. The second image is yielded by a mirror mounted above the unit. The lifetime of such a structure was $5 \cdot 10^{-3} \mathrm{~s}$, that is, it was 50 times longer than the electric pulse duration. This phenomenon is very interesting but it is not the subject of the present report. It should be stressed that radiation traces were registered by nuclear emulsions located at some distance (up to two meters) from the plasma structure.
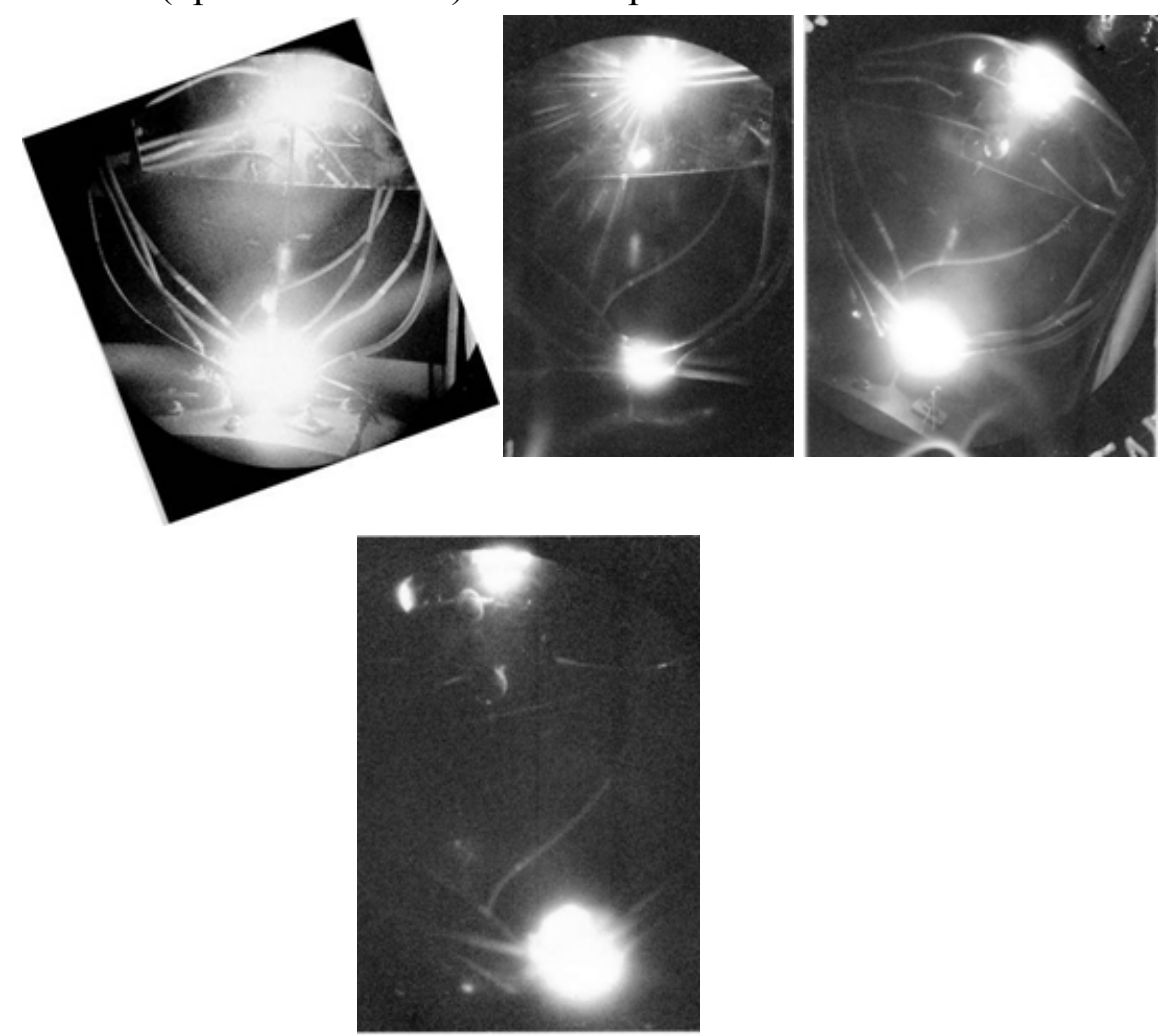

Figure 6. Pictures seen on the EOT screens. Exposure time 130 mcs. Time delay between the frames 1 ms. 
Typical traces are shown in Figure 7. The blackening density scale is shown in the figure. The traces are very unusual, and because of that the hypothetical radiation was called a 'strange' one. In Figure 8, a typical track created by an ion in a nuclear emulsion is shown for comparison. One can readily see that the traces we have found are much broader. Moreover, they are not continuous; frequently they are followed by narrower traces, and traces of $\delta$ electrons cannot be seen at all. Such traces (hairs) are always observed when high-energy particles are absorbed. This phenomenon has one more specific feature. Let $\vec{n}$ denote a vector perpendicular to the emulsion plane and $\vec{R}_{0}$ a radius-vector originating at the center of the unit. The traces are observed even in the configuration where $\vec{n}$ and $\vec{R}_{0}$ are collinear. We have experimentally found that the larger is the distance between the detector and the unit center, the narrower is the trace pattern. At a distance equal to about half meter, the track width is about $30 \mu \mathrm{m}$, while at a 2 -meter distance it is only around $5 \mu \mathrm{m}$.
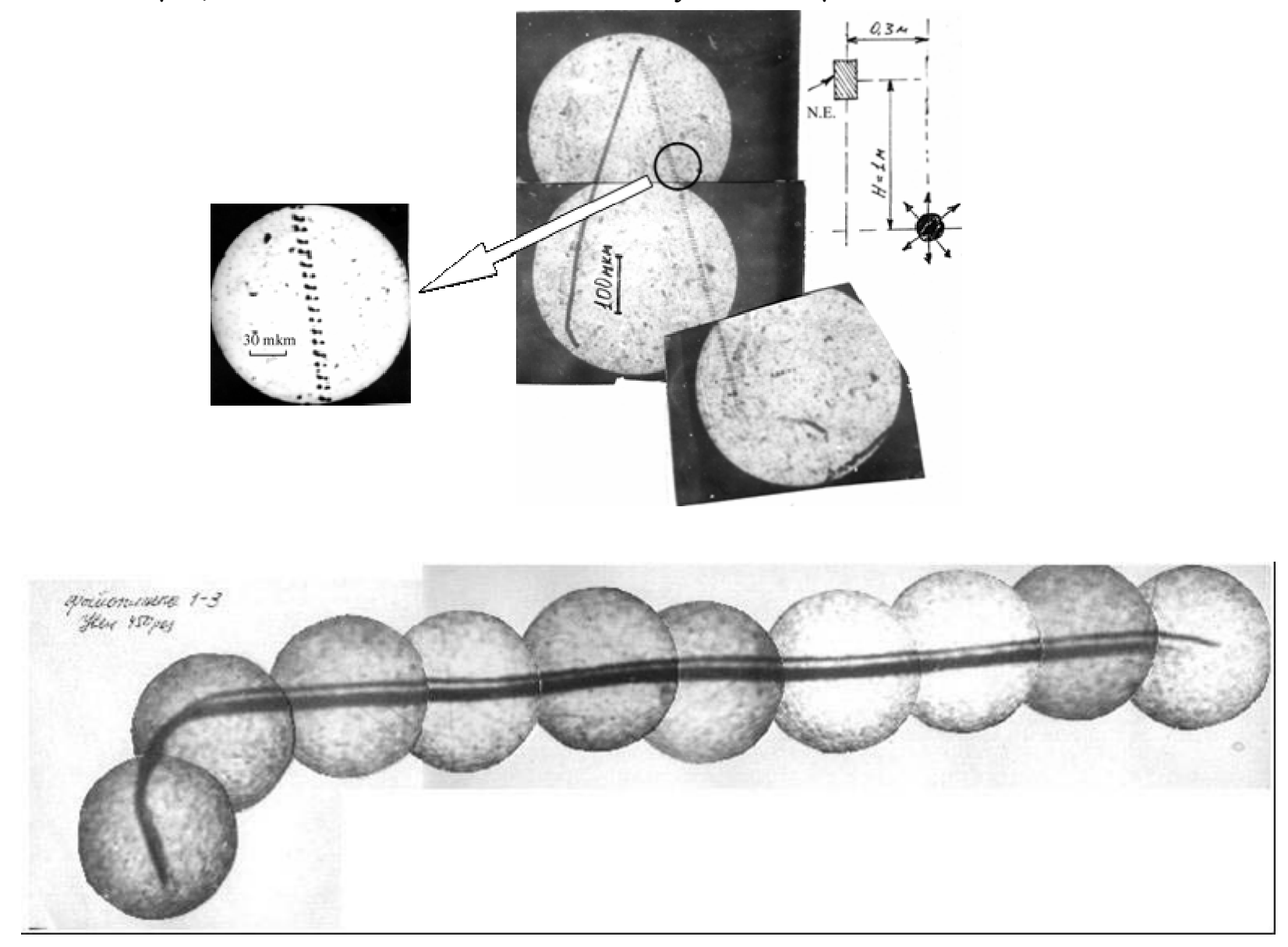

Figure 7. Typical traces. 


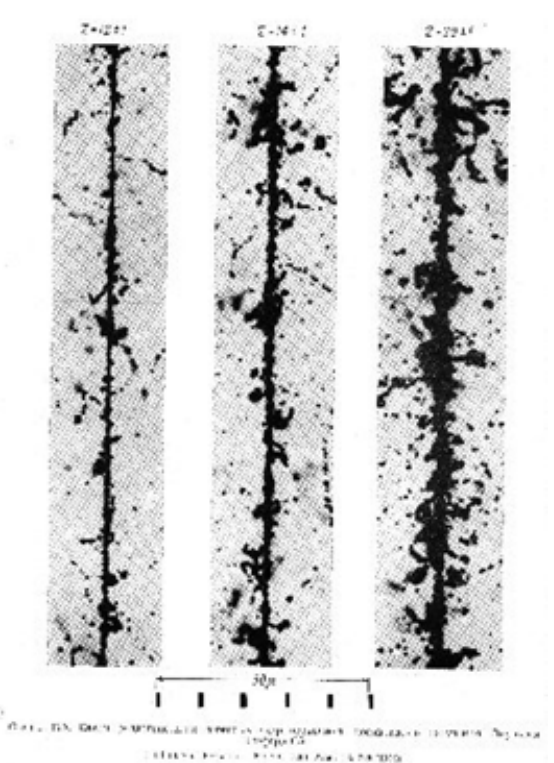

Figure 8.

Typical track created by an ion.

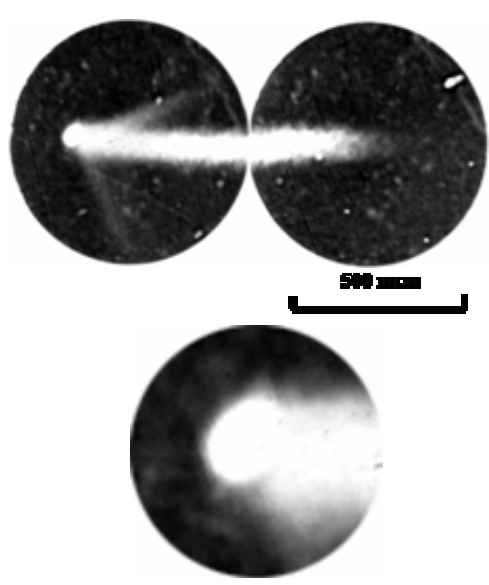

Figure 9.

Typical traces when the unit was subjected to a magnetic field.

To make sure that the traces are not related to some electromagnetic artifact, we installed detectors near the foil remnants only after the explosion. During 24 hours we were registering the traces which were indistinguishable from those, observed at the instant of electric pulse.

Thus, we have confirmed the nuclear origin of the radiation being registered. It should be noted that when the unit was subjected to a magnetic field [1], the traces in the nuclear emulsion changed. This is seen in the Figure 9.

Doctor Ivoilov will present in his report some very interesting results for the traces [8]. Here are some conclusions based on the presented experimental data.

1. The particle which left the trace in the nuclear emulsion is charged, as nuclear emulsions are insensitive to neutrons.

2. The particle cannot have electric charge, as otherwise it could not be able to pass through two meters of atmospheric air and two layers of black paper.

3. The particle does not have high energy, as no delta-electrons are observed.

4. The mechanism of the interaction between the particle and the photosensitive layer is not clear. Assuming the Coulomb mechanism, the absorbed energy estimated using the darkening area equals around $1 \mathrm{GeV}$.

5. The radiation is of nuclear origin; it interacts with magnetic fields.

This calls for a discussion of Lochak's magnetic monopole. Lochak created his theory 20 years before our experiments [9-12], that is, before those results for understanding and explaining of which we are now attempting to use it. It should be emphasized that this is a good omen for a theory. It is always suspicious when the theories are created specially to explain an experimental observation. They are like the circles drawn on a target after a shoot has been made.

Dirac's magnetic monopole theory is well known in physics [13]. In his theory Dirac with a mathematic ingenuity specific to him managed to relate non-integrability of the wave 
function's phase to the singularity which emerges when describing the interaction between the electron and the magnetic pole. Paradoxically, the Dirac monopole is not described by the fundamental equation of the quantum electrodynamics which is named after him. This seems to be the reason why the Dirac monopole is not in the mainstream of the development of theoretical physics. There is one more reason for that which is related to symmetry. Writing the equation for the Lorentz force acting on a magnetic charge:

$$
\overrightarrow{\mathrm{F}}_{\mathrm{L}}=\mathrm{g} \cdot\left(\overrightarrow{\mathrm{H}}-\frac{1}{\mathrm{c}} \cdot \overrightarrow{\mathrm{v}} \times \overrightarrow{\mathrm{E}}\right)
$$

we can see easily that inasmuch as $\overrightarrow{\mathrm{F}}$ is a polar vector and the right-hand side of the equation is a pseudo-vector, then $\mathrm{g}$, the magnetic charge, is to be a pseudo-scalar. This fact is very unusual for physicists. It means that the magnetic charge features a symmetry type other than the electric charge. Pierre Curie was the first to notice this fact. His reasoning was very simple and at the same time very wise. The electric charge is a scalar and it generates a field described by a polar vector $\mathrm{E}$. Inasmuch as the magnetic field vector $\mathrm{H}$ is a pseudo-vector, the source which generates this field (that is, the magnetic charge) should have the same symmetry, implying that the magnetic charge is a pseudo-scalar. Based on this Lochak argues: "There is no real symmetry between electricity and magnetism but there are two slopes of the same pinnacle: a vector slope and a pseudo-vector one."

In mathematical terms, the Dirac equation is invariant to two and only two gauge transformations. Indeed, the Dirac equation for a relativistic charged particle with spin $S=1 / 2$ in an external field can be written in the following form

$$
\left(\gamma_{\mu} \nabla_{\mu}+\frac{\mathrm{mc}}{\hbar}\right) \psi=0
$$

where $\gamma_{\mu}$ are $4 \times 4$ Dirac matrices, and $\psi$ is a bi-spinor.

Then, if we subject the bi-spinor to the following transformation

$$
\psi \rightarrow \psi^{\prime}=\exp \left(\mathrm{i} \frac{\mathrm{e}}{\hbar \mathrm{c}} \cdot \mathrm{I} \cdot \varphi\right) \cdot \psi
$$

where $\mathbf{I}$ is the unit matrix and the operators have the following form

$$
\begin{aligned}
& \nabla_{\mu}=\partial_{\mu}-\mathrm{i} \frac{\mathrm{e}}{\hbar \mathrm{c}} \cdot \mathrm{I} \cdot \mathrm{A}_{\mu}, \\
& \mathrm{A}_{\mu} \rightarrow \mathrm{A}_{\mu}+\partial_{\mu} \varphi,
\end{aligned}
$$

then Equation (1) transforms into the well-known equation for the electron:

$$
\gamma_{\mu}\left(\partial_{\mu}-\mathrm{i} \frac{\mathrm{e}}{\hbar \mathrm{c}} \mathrm{A}_{\mu}\right) \psi+\frac{\mathrm{mc}}{\hbar} \psi=0
$$

Now, if we use Dirac equation (1) without the mass term, that is, actually the equation describing the neutrino

$$
\gamma_{\mu} \nabla_{\mu} \psi=0
$$

and represent the gauge invariance using a pseudo-scalar matrix

$$
\gamma_{5}=\gamma_{1} \cdot \gamma_{2} \cdot \gamma_{3} \cdot \gamma_{4}=\left(\begin{array}{ll}
0 & \mathrm{I} \\
\mathrm{I} & 0
\end{array}\right),
$$

where $\mathbf{I}$ is the $2 \times 2$ unit matrix, and transform the bi-spinor as follows 


$$
\psi \rightarrow \psi^{\prime}=\exp \left(\mathrm{i} \frac{\mathrm{g}}{\hbar \mathrm{c}} \cdot \gamma_{5} \cdot \chi\right) \cdot \psi,
$$

then the operator will have the form

$$
\nabla_{\mu}=\partial_{\mu}-\frac{\mathrm{g}}{\hbar \mathrm{c}} \gamma_{5} \mathrm{~B}_{\mu}
$$

and the local gauge will have the following form

$$
\mathrm{B}_{\mu} \rightarrow \mathrm{B}_{\mu}+\mathrm{i} \cdot \partial_{\mu} \chi \text {. }
$$

Note that because of the pseudo-scalar matrix $\gamma_{5}$ the field $\mathbf{B}$ is a pseudo-potential and $\chi$ is a pseudo-phase. However, the pseudo-scalar feature of magnetism is expressed using the charge operator $\mathrm{G}=\mathrm{g} \cdot \gamma_{5}$ where $\mathrm{g}$ is a usual scalar. The Lochak equation for the magnetic charge has the form

$$
\gamma_{\mu}\left(\partial_{\mu}-\frac{\mathrm{g}}{\hbar \mathrm{c}} \gamma_{5} \mathrm{~B}_{\mu}\right) \psi=0 .
$$

The phase gauge using $\gamma_{5}$ was known before Lochak's works, but he managed to find the physical sense in Equation (4): it is the equation describing the magnetic monopole with zero mass. Thus, in Lochak's theory the magnetic monopole is a kind of the magnetically excited neutrino. Do not forget that it is described by the same equation as the neutrino. No doubt, Lochak's theory is not complete yet and needs further development. Professor Harald Stumpf has worked actively for several years to apply it to solve the exact physical problems and to integrate it into the Standard Model [14].

One might ask: If Lochak's theory is correct, why has it garnered so little interest for twenty years? The answer is obvious. French physicists are deeply convinced that all genuine physical theories can only be developed outside France. Other physicists usually do not read scientific publications in French. Because of that Lochak's works are not known to academics.

A more profound reason is that the Standard Model does not need the leptonic magnetic monopole. Today's physics are dominated by the dictatorship of democracy. Let us explain that using a simple example. About five years ago a CERN paper was published which had around 600 authors. The list of authors was longer than the article itself. As to the authors of the present report, we believe that a new idea may come to one head or at most two heads, but in no way to 600 heads at the same time. Bearing in mind that theorists and experimentalists tend to consider the Standard Model impeccable (something like a holy icon), you will understand the attitude to Lochak's theory. After this brief digression we should return to the main subject and draw some conclusions which are much needed by an experimentalist.

According to Lochak, the magnetic monopole is a fermion. Moreover, it features chiral symmetry [11]; under a spatial reflection the charge seems to change its sign. In reality the charge operator $G$ passes from one of its own meanings (for example corresponding to the left monopole) to another one (right monopole) [12]. This is very unusual for experimentalists who are accustomed to deal with electric charges which do not allow themselves such a behavior. According to the classification adopted in the elementary particle physics, the monopole is a lepton and, hence, it participates in weak interactions. Because its rest mass is zero, not much energy is needed to create a monopole-antimonopole pair. On the other hand, for this reason it is difficult to detect. Naturally one can ask: if everything is so easy, why 
hasn't the monopole been detected earlier? We believe that first it is necessary to find the monopole to make sure it exists, and only after that, will it be possible to answer this question.

Our experimental search for the monopole started with an attempt to capture it in a ferromagnetic trap. Foil made of ${ }^{57} \mathrm{Fe}$ was used in the trap. The idea was that capture of the monopole by the ferromagnetic will change the field on the ${ }^{57} \mathrm{Fe}$ nucleus. This in turn will affect the width of Mössbauer spectrum lines. Let (Fig. 10a) be the line width before irradiation. We would expect to see (b), and have instead observed (c). This means that the Mössbauer spectrum of ${ }^{57} \mathrm{Fe}$ (containing 6 lines) has not broadened but has only shifted by the same value equal to $\Delta \mathrm{H}=500 \pm 70$ Gs. This means that we observe some collective effect. Doctor Ivoilov will discuss this subject in detail in his report [15].

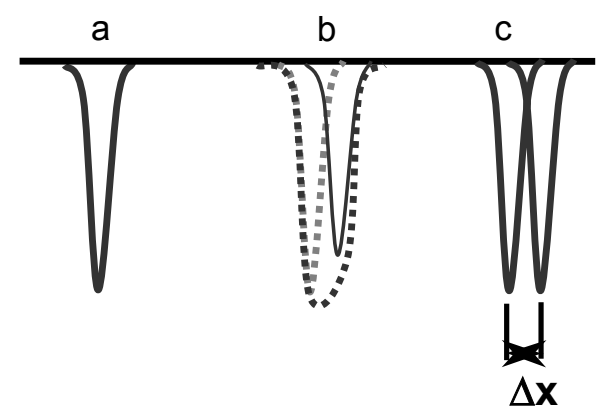

Figure 10.

Mössbauer spectrum

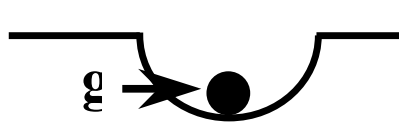

a

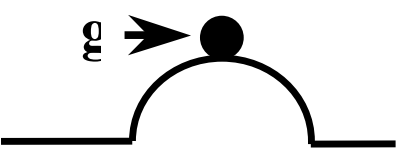

b

Figure 11.

System in stable (a) and unstable (b) position of equilibrium

Another feature of the magnetic monopole, which follows from Lochak's theory, is that it has a leptonic origin. Because of this, one can hope that the presence of the magnetic monopole affects the $\beta$-decay. Based on this, in the experiments with uranium salts we measured very accurately the $\beta$-decay periods of daughter isotopes in the uranium and thorium series. In the experiment, we have found that thorium secular equilibrium and, hence, the probability of the $\beta$ - decay of the protactinium isomer is strongly changed. Prof. Rukhadze will tell about our observations in his report [16].

The Lochak magnetic 'neutrino' has zero rest mass and, hence, it cannot exert any energetic effects on an atomic system. However, if a system rich in energy is in a nonequilibrium state and a chain reaction develops in it, the situation changes. This is shown in Fig. 11. In case (a) a small perturbation will only result in small oscillations. Theorists believe 
this is due to zero oscillations of vacuum. It is obvious that in case (b) the result will be quite different. A nuclear reactor is a good example of such system. Henri Rukhadze will describe in detail how catastrophic will be the consequences of even insignificant changes in the $\beta$ decay periods of nuclei [16].

Ammonium nitrate is another example of a system rich in energy [17]. Henri Lehn, our French colleague, will describe results of our joint experiments, held in our experimental unit, in which we observed interaction between magnetic charges and ammonium nitrate.

For understandable reasons the issues related to the interaction between the magnetic radiation and biological objects are of no small importance for us, because a human body is, to large extent, an electromagnetic system. Chelyabinsk biophysicists conducted some pilot experiments, in which laboratory animals were irradiated in our experimental unit. Doctor Pryakhin will describe the results of these experiments [18]. I will only mention that we have observed that the number of stem cells in the animals' marrow changes.

\section{Conclusions}

To summarize, we seem to have found a new type of interaction. It has magnetic nature and catalyzes nuclear processes by initiating weak interactions. In our opinion, a new type of activity has been found (g-or m-activity). It should be stressed that last statements are nothing but hypotheses though, in our opinion, they are not unsubstantiated. I would like to state on both Lochak's part and my own that should the aforementioned hypothesis prove to be wrong, we will bear scientific responsibility. If the result is positive, it will undoubtedly be an achievement of the entire French and Russian team. Even if the statements are not wrong, a lot of effort will be needed to check their correctness.

The results presented in this talk were obtained by a large team of specialists and technical staff. It is my pleasure to list the team members:

"RECOM" specialists: Dr. Dmitry Filippov, Dr. Vladimir Liksonov, Dr. Valery Kuznetsov, Dp.Ing. Alexander Govorun, Dp.Ing. Anatoly Volkovich, Dp.Ing. Sergei Smirnov, Dp.Ing. Victor Korolev, Dp.Ing. Alexander Guliaev, Dp.Ing. Chermen Kaitukov, Dp.Ing. Victor Kalensky, Dp.Ing. Sergei Petrushko.

"RECOM" technical staff: Vladimir Shevchenko, Pavel Strashko, Dp.Ing. Tatiana Sokolova, Nikolai Zubkov, Alexander Gaverdovsky, Vladimir Bajushkin, Evgeny Sergeev, Vladimir Petrushko.

National Research Center "Kurchatov Institute": Pr.ph. Vladlen Tsinoev, Dp.In. Sergei Zukov, Dr. Rosa Rjabova, Dr. Jury Dontsov, Dp.Ing. Boris Novoselov, Dr. Leonid Elesin, Dp.Ing Vladimir Dorovskoi, Dr. Sviatoslav Demkin, Dp.Ing. Vladimir Stolyarov.

Institute of Inorganic Chemistry: Dr. Alexander Steblevsky.

Central Research Institute for Chemical Machine Building: Dr. Pavel Stolyarov.

Institute of General Physics: Professor Anri Rukhadze.

Kazan State University: Dr. Nikolai Ivoilov.

Chelyabinsk State University: Dr. Evgeny Pryakhin.

Institute of Chemical Physics: Dr. Vladimir Fedotov.

Many observations have not been included in this report because of lack of space.

\section{References:}

1. L.I. Urutskoev, V.I. Liksonov, V.G. Tsinoev // Observation of transformation of chemical elements during an electric discharge // Ann. Fond. L.de Broglie, 2002, v. 27, p. 701.

2. V.D. Kunznetsov, G.V. Mishinsky, F.M.Penkov, V.I.Arbuzov, V.I.Zhemenik // Low Energy Transmutation of Atomic Nuclei of Chemical Elements // Annales de la Fondation Louis de Broglie, 2003, v. 28, \# 2, p. 173-213.

3. A.G. Volkovich, A.P. Govorun, A.A. Gulyaev, S.V. Zhukov, V.L. Kuznetsov, A.A. Rukhadze, A.V. Steblevskii, L.I. Urutskoev. // Experimental observation of the distortion of the uranium isotopic relationship and violation of the thorium-234 secular equilibrium upon electric explosion // Bulletin Of The Lebedev Physics Institute, 2002, \#8. 
4. V.M. Dorovskoi, L.A. Elesin, D.V. Filippov, A.V. Steblevskii, V.L. Stolyarov, L.I. Urutskoev // Electron microscopy study of the transformation products // IEEE Transactions on Plasma Science (to be published)

5. A.A. Rukhadze, L.I. Urutskoev, D.V. Filippov // On the possibility of low-energy nuclear reactions from the viewpoint of conservation laws // Bulletin Of The Lebedev Physics Institute, 2004, \#4.

6. L.I. Urutskoev, D.V. Filippov // On the possibility of low-energy nuclear reactions in low energy plasma from the viewpoint of conservation laws // Moscow, NIZ GTP Erzion, 2003, p. $84-97$.

7. D.V. Filippov, A.A. Rukhadze, L.I. Urutskoev // Effects of atomic electrons on nuclear stability and radioactive decay // Report, ICCF 11, Marseille 2004.

8. Ivoilov N.G. // Low Energy Generation Of The "Strange" Radiation // Report, ICCF 11, Marseille 2004.

9. G. Lochak, Annales de la Fondation Louis de Broglie, 8, p. 345, 1983; 9, p. 5, 1984.

10. G. Lochak, Wave equation for a magnetic monopole, International Journal for Theoretical Physics, 24, p. 1019, 1985.

11. G. Lochak, The symmetry between Electricity and Magnetism and the problem of the existence of magnetic monopoles, in Advanced Electromagnetism, ed. T.W. Barrett, D.M. Grimes, World Scientific, Singapore, pp. 105-148, 1995.

12. G. Lochak // Leptonic monopole and weak interactions // Report, ICCF 11, Marseille 2004.

13. Dirac P.A.M. // Proc. Roy. Soc., A133, 60 (1931); Dirac P.A.M. // Phys. Rev., 74, 817 (1948).

14. H. Stumpf // Simultaneous formation of electric and magnetic photon states by electroweak symmetry breaking // Z. Naturforsch. 59a, 185-195, 2004.

15. N.G. Ivoilov, L.I. Urutskoev // The influence of "strange" radiation on Mössbauer spectrum of $\mathrm{Fe}^{57}$ in metallic foils // Prikladnaya Fizika (Applied Physics; in Russian), 2004, \#5. p. 20.

16. A.A. Rukhadze, L.I. Urutskoev, D.V. Filippov // On the possible magnetic mechanism of shortening the runaway of RBMK-1000 reactor at Chernobyl Nuclear Power Plant // Bulletin Of The Lebedev Physics Institute, 2004, \#1.

17. H. Lehn // Report, ICCF 11, Marseille 2004.

18. E.A. Pryakhin, L.I. Urutskoyev, G.A. Tryapitsina, A.V. Akleyev // Assesment of the Biological Effects of "Strange" Radiation // Report, ICCF 11, Marseille 2004. 